Revue internationale P.M.E.

Économie et gestion de la petite et moyenne entreprise

\title{
L'épargne informelle et le financement de l'entreprise productive : référence spéciale aux tontines et à l'artisanat béninois
}

\author{
Simon C. Gnansounou
}

Volume 5, numéro 3-4, 1992

URI : https://id.erudit.org/iderudit/1008153ar

DOI : https://doi.org/10.7202/1008153ar

Aller au sommaire du numéro

Éditeur(s)

Presses de l’Université du Québec

ISSN

0776-5436 (imprimé)

1918-9699 (numérique)

Découvrir la revue

Citer cet article

Gnansounou, S. C. (1992). L'épargne informelle et le financement de l'entreprise productive : référence spéciale aux tontines et à l'artisanat béninois. Revue internationale P.M.E., 5(3-4), 21-47.

https://doi.org/10.7202/1008153ar

\section{Résumé de l'article}

Dépassant le niveau descriptif du phénomène tontinier au Bénin, l'auteur a testé la capacité réelle de financement des tontines au regard des activités productives et ce, à partir d'une population nationale cible composée de 1620 entreprises individuelles artisanales.

Il s'agit en effet d'une étude fort complète, menée en termes à la fois quantitatifs et financiers et conçue d'une part à partir de l'association entre l'activité économique de l'artisan et l'individu ménage, et d'autre part, à partir de la liaison entre la participation de cet individu aux tontines et le financement de l'activité qu'il réalise.

L'analyse des données recueillies a permis deux observations intéressantes :

- Seulement $42,48 \%$ des ressources tontinières mobilisées ont servi à assurer $25 \%$ des financements, avec la précision que les financements effectivement réalisés - toutes sources confondues - ne représentent que $65,55 \%$ des besoins exprimés.

- Le facteur temps, non intégré dans cette sorte d'intermédiation financière informelle, enlève aux fonds prêtables que sont les ressources tontinières tout rôle de contrainte et de stimulant pour une gestion efficiente de la microentreprise. Faut-il pour autant ne fonder aucun espoir sur cette explosion de l'offre informelle de services financiers qu'assure la tontine? La perfectibilité qu'offre la souplesse du système tontinier autorise l'optimisme. 


\title{
L'épargne informelle et le financement de l'entreprise productive : référence spéciale aux tontines et à l'artisanat béninois
}

\author{
Simon C. GNANSOUNOU*
}

Université nationale du Bénin

\begin{abstract}
RÉSUMÉ
Dépassant le niveau descriptif du phénomène tontinier au Bénin, l'auteur a testé la capacité réelle de financement des tontines au regard des activités productives et ce, à partir d'une population nationale cible composée de 1620 entreprises individuelles artisanales.

II s'agit en effet d'une étude fort complète, menée en termes à la fois quantitatifs et financiers et conçue d'une part à partir de l'association entre l'activité économique de l'artisan et l'individu ménage, et d'autre part, à partir de la liaison entre la participation de cet individu aux tontines et le financement de l'activité qu'il réalise.

L'analyse des données recueillies a permis deux observations intéressantes:

- Seulement 42,48 \% des ressources tontinières mobilisées ont servi à assurer $25 \%$ des financements, avec la précision que les financements effectivement réalisés - toutes sources confondues - ne représentent que 65,55\% des besoins exprimés.

- Le facteur temps, non intégré dans cette sorte d'intermédiation financière informelle, enlève aux fonds prêtables que sont les ressources tontinières tout rôle de contrainte et de stimulant pour une gestion efficiente de la microentreprise.
\end{abstract}

Faut-il pour autant ne fonder aucun espoir sur cette explosion de l'offre informelle de services financiers qu'assure la tontine? La perfectibilité qu'offre la souplesse du système tontinier autorise l'optimisme.

* Simon C. Gnansounou est actuellement enseignant à l'Université nationale du Bénin. Il est titulaire d'un doctorat nouveau régime en sciences économiques obtenu à l'Université d'Orléans. En matière de recherche, il s'intéresse aux problèmes de financement dans le secteur informel et à l'approche managériale de la conduite de l'entreprise en Afrique. Adresse : B.P. 03-2236, Cotonou, Bénin. 


\begin{abstract}
After a short description of tontines in Benin, the author of this paper presents the results of a survey on their capacity to finance economic activity. The survey was conducted with 1,620 workshops owned by sole traders.

The paper provides quantitative and financial information on the activity of craftsmen as economic agents and heads of households on the one hand, and as lenders to or borrowers from tontines on the other hand.

Two major observations stand out :

- only $42,48 \%$ of all the resources collected by tontines covered $25 \%$ of the workshop's financial needs ;

- as the time factor is not taken into consideration in this type of informal financial transaction, tontine funds-lending offers no incentive towards increased efficiency in the management of small businesses.

Yet, one may not conclude that the growing supply of informal financial services from tontines falls on barren soil. The very flexibility of tontines provides reason for hope.
\end{abstract}

\title{
RESUMEN
}

El autor no se ha limitado al nivel descriptivo del fenómeno de las tontinas en Benin, sino que además ha comprobado la capacidad real de financiación de las tontinas en cuanto a las actividades productivas a partir de una población nacional compuesta por 1,620 empresas individuales artesanales.

En efecto, se trata de un estudios concebido a partir de la asociación entre la actividad económica del artesanado y el individuo, y a partir de la relación entre la participación de este individuo en las tontinas y la financiación de la actividad que éste realiza.

El análisis de datos obtenidos ha permitido dos observaciones interesantes :

- Solamente el $42,48 \%$ de los recursos de los suscriptores de tontinas mobilizados han servido para asegurar un $25 \%$ de financiaciones.

- El factor tiempo, no integrado en este tipe de intermediación financiera informal, les suprime el papel de obligación, y de estímulo para una gestión eficaz de la microempresa, a los fondos prestables que constituyen los recursos procedentes de las tontinas.

A pesar de ello, el crecimiento de la oferta informal y la flexibilidad del sisteme de las toninas inducen al timismo. 


\section{Introduction}

Le constat est aujourd'hui général. Le marasme dans lequel s'enfonce de plus en plus le continent africain en général et sa partie sub-saharienne en particulier, dont fait partie le Bénin, favorise l'expansion de l'économie non structurée désormais désignée, avec ou sans nuance par les économistes, sous la thématique du « secteur informel ${ }^{1} »$.

Au Bénin, ce secteur est très développé et se caractérise par la prolifération physique de petites activités économiques très diversifiées évoluant hors des cadres légaux, sans avantages sociaux ni protection d'aucune sorte.

En effet, loin des structures officielles et des circuits organisés et «modernes », les travailleurs du secteur informel, marginaux, généralement dédaignés par les pouvoirs centraux, ne figurent qu'incidemment dans les recensements officiels. Pourtant, selon les estimations du Bureau international du travail (BIT), ces « laissés-pour-compte du développement » constituent 30 à $70 \%$ de l'activité urbaine dans le tiers monde. Selon cet organisme, ce chiffre au Bénin était de $66,66 \%$ en 1986 et ne fait qu'augmenter d'année en année. En effet, sur 210000 travailleurs en avril 1986, 140000 individus regroupés dans 87000 entreprises évoluaient dans le secteur informel, tandis que le secteur formel n'employait environ que 70000 personnes $^{2}$.

La devise au sein du secteur informel est avant tout « s'unir pour survivre ", ce qui empêche les mécanismes et le rationalisme de la science économique de jouer effectivement. Il n'en demeure pas moins, d'une part, que ce secteur reste globalement la soupape de sûreté du système économico-social du tiers monde, et d'autre part (au niveau du sous-secteur informatique que constitue l'artisanat), qu'il représente la structure à prendre en compte et à améliorer pour la mise en place progressive d'un tissu économique performant.

C'est pourquoi, dans notre recherche de secteurs productifs (donc bénéfiques) à financer par la tontine, notre choix a porté sur les activités artisanales avec l'idée sous-jacente que l'économie informelle est la mieux placée pour être financée par la finance informelle.

À l'étape actuelle de la réflexion, nous ne reviendrons plus sur les formes et les mécanismes de fonctionnement des tontines béninoises, ni sur les conditions détaillées de réalisation de nos investigations dans l'artisanat

1. J.C. Martineau (1991 a et b) et C. Miras (1990).

2. BIT-PNUD-Projet BEN/86/005 (1987) : une version préliminaire, plus complète, est parue sous le même titre, dans les Notes de recherche du Réseau entrepreneuriat de I'UREF $\left(n^{\circ} 91-20\right)$. 
béninois ${ }^{3}$. Nous nous contenterons de présenter globalement ces enquêtes à travers les résultats nationaux obtenus tout en procédant à l'analyse financière et économique du financement des activités artisanales à partir des ressources tontinières.

\section{Les enquêtes}

Les enquêtes se sont déroulées d'octobre 1989 à mars 1990 et ont porté sur 1620 entreprises artisanales, à raison de 270 entreprises par département ${ }^{4}$.

En tant que première investigation à dimension nationale sur le couple informel "tontine-artisanat ", ces enquêtes ne se sont pas déroulées sans difficultés, des difficultés qui ont été de trois ordres.

La première difficulté est relative au milieu informel béninois qui est resté fermé, à cause des intellectuels que sont les chercheurs, et aussi de la barrière linguistique qui le sépare parfois des enquêteurs. Pour contourner cette difficulté, que nous avions du reste pressentie depuis la phase des pré-enquêtes, nous avons eu recours à trois stratagèmes :

- Un appel a été fait aux DPCAT, en l'occurrence à leur service chargé de l'artisanat pour nous servir de guide auprès des artisans.

- Le recrutement des enquêteurs a chaque fois été fait sur place en tenant compte de leur connaissance du monde local des artisans (fils ou parents directs d'artisans) et en tenant compte d'un niveau intellectuel minimum équivalant au brevet élémentaire.

- La dernière solution a consisté à dire aux artisans que les enquêtes ont été demandées par un groupe financier qui compte financer les activités artisanales dans le pays en $y$ installant très prochainement une Banque de l'Artisanat.

La deuxième difficulté à laquelle nous ayoons été confrontés se rapporte à la non-participation (ou la cessation) de certains artisans à la tontine. Ils ont été au nombre de 67 et il a fallu remplacer chacun d'eux par un collègue travaillant sur le même produit artisanal et nécessairement membre d'au moins une tontine.

3. Toutes les informations relatives à ces deux aspects du travail sont contenues dans Gnansounou, (1991 a et b).

4. Le Bénin compte six départements : l'Atacora, l'Atlantique, le Borgou, le Mono, l'Ouémé et le Zou.

5. DPCAT : Direction provinciale du commerce, de l'artisanat et du tourisme. 
Cette substitution n'a pas été toujours facile et il nous a fallu descendre dans les quartiers lointains ou les villages proches pour sauvegarder notre échantillon de base.

Le troisième problème fut d'ordre financier et vient du fait que les artisans ont eu tendance, surtout dans le Borgou, à exiger une rémunération contre les renseignements à livrer ou, tout au moins, un achat conséquent de produits artisanaux par les enquêteurs. Il n'a pas été rare de débourser $200 \mathrm{~F}$ (don) à $500 \mathrm{~F}$ (achat) afin d'interroger un artisan et ceci en déduction de nos propres per diems de recherche, aucun fonds n'ayant été prévu à cet effet.

Toutes ces précautions n'ont malheureusement pas suffi à éviter à nos investigations certaines faiblesses que nos premiers dépouillements ont mises à nu. Ces faiblesses se sont manifestées en termes d'incohérences dont voici les principales :

- Certains artisans prétendent avoir des problèmes de financement de leurs activités alors que les ressources financières mobilisées dépassent leurs besoins de financement exprimés.

- Parce que «informés » de la possibilité d'installation d'une banque pour les entreprises artisanales, les artisans pouvaient exagérer le montant de leurs besoins en financement et de leur chiffre d'affaires.

- La méfiance des artisans explique certainement leur déclaration presque uniforme $(74,39 \%)$ d'appartenir à une seule tontine ; histoire de cacher une partie de leurs ressources tontinières aux enquêteurs.

- Deux entreprises artisanales déclarent avoir eu recours aux banques pour leur financement, mais le montant de ces financements bancaires est égal à 0 .

Il nous a donc fallu éliminer ou atténuer au départ les incohérences des enquêtes avant de procéder à l'agrégation des données et à la confection des tableaux relatifs aux résultats globaux. Ce fut un travail très fastidieux qui nous a pris plusieurs mois.

C'est à partir de ces résultats globaux que le présent document essaie d'abord de présenter les problèmes et les sources de financement de l'artisanat béninois par rapport aux ressources tontinières. Il essaie ensuite, d'une manière plus spécifique, de rendre compte des coûts du recours aux ressources tontinières par les entreprises artisanales. 


\section{Problèmes et sources de financement de l'artisanat béninois et le rôle des ressources tontinières}

La gestion d'une entreprise, qu'elle soit de l'informel ou du secteur officiel, nécessite la mobilisation de trois types de ressources:

- les ressources humaines;

- les ressources matérielles et techniques, et

- les ressources financières.

Toutes ces ressources, aussi importantes les unes que les autres, peuvent se traduire en termes de difficultés, difficultés que l'on peut mesurer à partir de taux et de ratios précis.

\subsection{Les difficultés des entreprises artisanales béninoises : l'acuité des problèmes financiers}

Partant du principe que toutes les ressources se valent dans une entreprise, nous les avons exprimées en termes de difficultés en les « éclatant » en 19 problèmes à résoudre. Seuls 8 artisans sur les 1620 , soit $0,49 \%$ de l'échantillon prétendent n'avoir aucune difficulté.

\subsubsection{La hiérarchisation des difficultés des entreprises artisanales béninoises}

Les problèmes les plus cruciaux de l'artisanat béninois se présentent comme suit, par ordre décroissant :

- financement de l'activité ;

- logement ou installation de l'atelier ;

- matériels et outillages;

- matières premières ;

- tracasseries familiales et sorcellerie ;

- déplacement et transport ;

- recouvrement des créances ;

- écoulement du produit. 
Toutes ces difficultés méritent attention et apport de solution; mais compte tenu de notre cadre de réflexion essentiellement axé sur les problèmes de financement, nous insistons sur le fait que les plus graves problèmes rencontrés par les artisans sont d'ordre financier et concerne $96,60 \%$ des artisans interrogés.

Comment se traduit dans les faits cette difficulté de financement?

\subsubsection{Niveau de résolution des difficultés financières dans l'artisanat béninois}

Le taux de couverture des besoins de financement (TCBF) de l'artisanat béninois est globalement de $65,55 \%$, ce qui signifie que $34,45 \%$ de ces besoins ne sont pas satisfaits, difficultés inégalement supportées par les différents secteurs de l'artisanat. Ce sont les « artisans de service " qui ont la meilleure couverture de financement $(\mathrm{TCBF}=68,06 \%)$, contre $66,15 \%$ pour les « artisans de production» et $47,84 \%$ pour « les artisans d'art et de décoration». Ceci dégage des taux ${ }^{6}$ de recherche de financement (TRF) respectifs de $31,94 \%, 33,85 \%$ et $52,16 \%$, montrant que c'est l'artisanat d'art et de décoration qui est le secteur le plus touché par les problèmes de financement. Cette situation implique que le troisième secteur artisanal est le plus susceptible d'avoir recours aux fonds propres pour faire face à ses problèmes de financement. Ceci le rend plus productif avec un taux de profit de $143,24 \%$, contre $130,61 \%$ pour les artisans de services et $43,71 \%$ pour les artisans de production.

Ces taux de profit mettent en évidence non seulement le caractère globalement rentable des activités artisanales au Bénin, mais également une productivité $(\mathrm{PK})$ très élevée du capital dans ce secteur (PK compris entre $143,71 \%$ et $243,24 \%$ ). D'où proviennent alors ces capitaux performants, mais insuffisants, qui alimentent la production artisanale béninoise?

\subsection{Les sources de financement de l'artisanat: priorité aux aides interpersonnelles et aux ressources tontinières}

L'hypothèse de base retient que, faute des ressources officielles bancaires, l'artisanat est contraint d'utiliser les ressources informelles tontinières ${ }^{7}$; mais nos investigations ont abouti à deux constats fondamentaux en la matière :

6. $\mathrm{TRF}=100 \%-\mathrm{TCBF}=$ taux de recherche de financement.

7. F. Vincent (1986, p. 4) disait déjà que « le meilleur moyen de financer le développement local est d'utiliser l'épargne des tontines ou des caisses de solidarité qui existent ». 
- Les aides interpersonnelles et les ressources tontinières semblent rivaliser en importance.

- Les ressources tontinières ne financent pas prioritairement l'artisanat.

\subsubsection{Primauté des ressources de solidarité : les tontines, prolongement des aides interpersonnelles}

Le financement d'une entreprise individuelle repose sur l'apport de son propriétaire. Dans le cas d'une entreprise artisanale, cet apport doit être assuré par l'artisan qui dispose au Bénin de certaines possibilités particulières dont l'utilisation se présente comme suit (en termes de fréquence d'utilisation) :

1) Ressources tontinières $\ldots \ldots \ldots \ldots \ldots \ldots \ldots \ldots \ldots \ldots \ldots \ldots \ldots \ldots, 08 \%$

2) Aides interpersonnelles (parents et amis) ...........26,36\%

3) Réserves et autofinancement $\ldots \ldots \ldots \ldots \ldots \ldots \ldots \ldots \ldots, 51 \%$

4) Avances sur commande (clients) $\ldots \ldots \ldots \ldots \ldots \ldots \ldots \ldots \ldots$

5) État (avances et subventions) $\ldots \ldots \ldots \ldots \ldots \ldots \ldots \ldots \ldots \ldots \ldots$

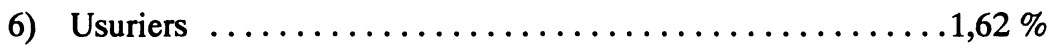

7) Mutuelles officielles $\ldots \ldots \ldots \ldots \ldots \ldots \ldots \ldots \ldots \ldots \ldots \ldots$

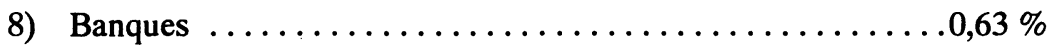

Ce classement montre que les « ressources de solidarité » que constituent l'épargne tontinière et les aides interpersonnelles sont les plus utilisées, avec une préférence relative pour les sources tontinières. Mais la capacité d'autofinancement du secteur n'est pas négligeable si l'on agrège les réserves ${ }^{8}$ et les avances consenties par les clients. Il est à remarquer que le très faible recours aux ressources officielles est patent (État, Mutuelles officielles et Banques) : dans ce domaine, la fréquentation des institutions bancaires est quasi nulle. Quant au faible taux de fréquentation des usuriers, il faudra le prendre avec beaucoup de réserve. En effet, si la tontine est aujourd'hui reconnue légale au Bénin, l'usure reste une activité interdite et réprimée par la loi. Il est par conséquent certain que les informations livrées par les artisans dans ce cadre ont été partielles. L'observation principale que nous pouvons faire à ce sujet est que, dans le système usurier béninois, " pourvoyeurs et receveurs de fonds se protègent mutuellement contre la loi et les curieux ». Il semblerait que la relative importance du volume des fonds provenant des aides interpersonnelles par rapport aux autres sources, s'explique en partie par le fait que les artisans

8. Il s'agit des fonds propres des entreprises artisanales. 
remplacent à dessein dans leur déclaration, « usuriers » par « parents et amis », gonflant artificiellement l'apport des aides interpersonnelles suivant l'ordre ciaprès (en termes de volume de fonds) :

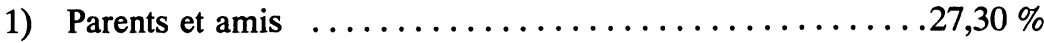

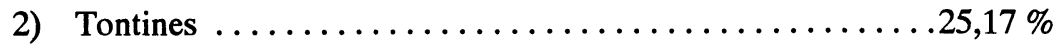

3) Avances sur commandes $\ldots \ldots \ldots \ldots \ldots \ldots \ldots \ldots \ldots \ldots \ldots$

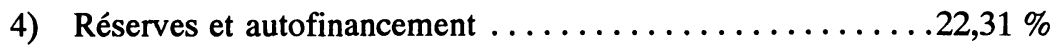

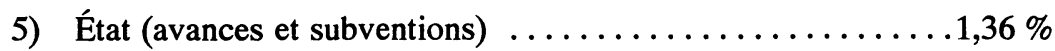

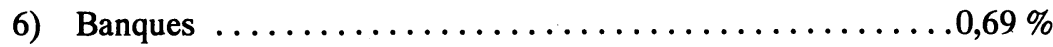

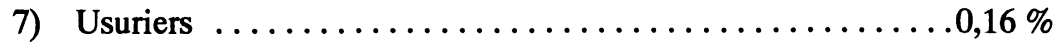

8) Mutuelles officielles $\ldots \ldots \ldots \ldots \ldots \ldots \ldots \ldots \ldots \ldots, 06 \%$

D'après la liste, l'usure n'assure que $0,16 \%$ des fonds de financement de l'artisanat béninois, ceci expliquant le surclassement peut-être anormal des aides interpersonnelles $(27,30 \%)$ par rapport aux ressources tontinières $(25,17 \%)$.

C'est au moment de l'évaluation du coût de l'argent dans le financement de l'activité artisanale que nous nous rendrons compte qu'il est anachronique de confondre « ressources usuraires », « aides interpersonnelles » et « ressources tontinières ». Toutefois, les aides interpersonnelles restent une réalité que la seule évocation des pratiques usuraires ne saurait effacer au Bénin.

En effet, les ressources tontinières ont pour première racine la solidarité entre les membres d'une même famille, d'un même clan, d'une même ethnie... Et dans ce schéma, l'usure n'est qu'une forme perverse de la solidarité interpersonnelle. En dernière analyse, ressources tontinières et aides interpersonnelles se complètent beaucoup plus qu'elles ne « rivalisent » entre elles.

Plusieurs études ont souligné cette importance de l'aide interpersonnelle dans le processus de financement des entreprises. Par exemple, Light (1972) rapporte que la pratique des clubs d'épargne explique les différents taux de représentation des groupes ethniques japonais, chinois, noir américain et noir des Caraỉbes dans le petit commerce aux États-Unis. Par ailleurs, Shelly Tenenbaum (1986) a étudié l'importance des sociétés philanthropiques de prêt juives (une institution juive traditionnelle) dans le financement des petits entrepreneurs juifs aux États-Unis durant une période où les banques n'accordaient pas de prêts aux petits emprunteurs, particuliers ou entreprises ${ }^{9}$. On remarque que le réseau intra-ethnique sert effectivement de source de financement à la

9. Cité par G.A. Brenner, H. Fouda et J.-M. Toulouse (1990, p. 3-4). 
micro-activité à travers les aides ou prêts directs et les tontines. Ces dernières représentent $28,08 \%$ des sources de financement. Mais ces sources tontinières sollicitées par $79,25 \%$ des artisans ne couvrent que $25,17 \%$ des financements réalisés par les entreprises artisanales. Cette relative faiblesse de l'apport financier des tontines peut s'expliquer de deux manières au Bénin :

- soit les ressources tontinières sont très faibles ;

- soit les ressources tontinières, bien qu'élevées, sont détournées à d'autres fins que le financement de la production artisanale.

\subsubsection{Les ressources tontinières : le financement non prioritaire de l'artisanat}

\section{Ressources tontinières et activités artisanales au Bénin}

Aussi paradoxal que cela puisse paraître, l'artisanat béninois, même face à la quasi-impossibilité de faire appel aux ressources bancaires, ne consacre pas la totalité de ses ressources tontinières à son financement, loin s'en faut. Les $25,17 \%$ du financement qu'elles assurent (742 millions de FCFA) ne représentent que $42,48 \%$ des ressources tontinières nettes disponibles (1746,5 millions de FCFA), ce qui dégage un taux global de détournement des ressources tontinières vers d'autres objectifs de $57,52 \%$. Cette structure de financement par rapport à l'épargne tontinière varie très peu d'un secteur d'activité artisanale à un autre, et se présente comme suit :

Financement artisanat Financement autres activités

- Artisans de production

- Artisans de service

- Artisans d'art et de décoration

Ensemble
$42,35 \%$

$42,94 \%$

$42,12 \%$

$42,48 \%$
$57,65 \%$

$57,06 \%$

$57,88 \%$

$57,52 \%$

Nous pouvons en déduire que, d'une façon globale, $57,5 \%$ des ressources tontinières ne servent pas à financer l'artisanat. Les $42,5 \%$ qui y sont consacrés le sont sous trois formes :

$$
\begin{array}{ll}
\text { - Avances } & 46,17 \% \\
\text { - Levées nettes } & 37,86 \% \\
\text { - Crédits } & 15,97 \%
\end{array}
$$

et suivant la répartition contrastée par secteur que voici : 


\begin{tabular}{lccc}
\hline & $\begin{array}{c}\text { Secteur I } \\
\text { (Production) }\end{array}$ & $\begin{array}{c}\text { Secteur II } \\
\text { (Service) }\end{array}$ & $\begin{array}{c}\text { Secteur III } \\
\text { (Art et décoration) }\end{array}$ \\
\hline - Avances & $44,89 \%$ & $53,82 \%$ & $28,35 \%$ \\
- Levées nettes & $37,45 \%$ & $31,16 \%$ & $71,20 \%$ \\
- Crédits & $17,66 \%$ & $15,02 \%$ & $0,45 \%$ \\
\hline
\end{tabular}

Cette répartition montre que les artisans n'ont pas le même comportement vis-à-vis des ressources tontinières : les artisans de production utilisent presque autant les avances tontinières que les levées nettes sans négliger pour autant les crédits tontiniers ; les artisans de service donnent une priorité relative aux avances suivies de la forme des levées à terme tout en ayant recours aux crédits ; quant aux artisans d'art et de décoration, ils n'ont pas recours aux crédits $(0,45 \%)$ et peu aux avances $(28,35 \%)$, mais se contentent souvent d'attendre la fin du cycle des tontines pour lever les fonds $(71,20 \%)$. Si les deux premiers secteurs se rapprochent en matière d'utilisation des ressources tontinières, c'est beaucoup plus à cause de leurs besoins de financement plus élevés que ceux de l'artisanat d'art et de décoration pour qui la tontine est d'abord un instrument d'épargne avant d'être un moyen de crédit et d'investissement. En effet, le secteur III reste encore très proche du monde traditionnel béninois qui n'est pas encore habitué à financer les activités à partir des crédits ${ }^{10}$.

Après cette rapide digression sur les formes d'utilisation des ressources tontinières dans l'artisanat béninois, force nous est de nous demander à quoi servent effectivement les $42,48 \%$ de ces ressources qui y sont consacrées et les $57,52 \%$ de ces mêmes ressources qui n'y sont pas investies.

\section{Destinations des ressources tontinières des artisans béninois}

L'orientation que font les artisans de leurs ressources tontinières permet d'établir l'ordre de préférence suivant :

1) Financement de l'artisanat $\ldots \ldots \ldots \ldots \ldots \ldots \ldots \ldots .42,48 \%$

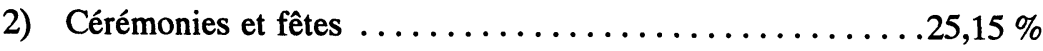

3) Terrain et construction $\ldots \ldots \ldots \ldots \ldots \ldots \ldots \ldots \ldots, 0 . \ldots \ldots$

4) Financement autres activités productives $\ldots \ldots \ldots \ldots \ldots 6,57 \%$

10. Jean-Noël Chartier du BIT, coordonnateur du Projet « Mutuelles officielles PNUDBIT », a fait la même remarque en proclamant : « Fait surprenant, il y a plus d'épargne versée que de crédits sollicités. Les artisans n'ont pas encore acquis le réflexe d'emprunter pour développer leur entreprise », J.C. Martineau (1991a, p.118). 
5) Thésaurisation et autres tontines $\ldots \ldots \ldots \ldots \ldots \ldots \ldots .3,27 \%$

6) Remboursement dettes artisanat $\ldots \ldots \ldots \ldots \ldots \ldots \ldots \ldots \ldots \ldots \ldots$

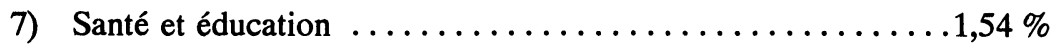

8) Remboursement autres dettes $\ldots \ldots \ldots \ldots \ldots \ldots \ldots \ldots \ldots, 43 \%$

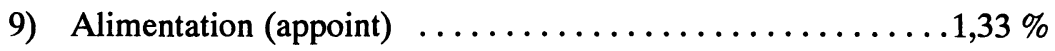

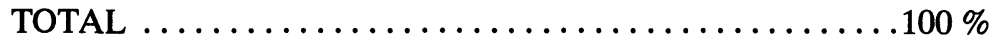

Cette répartition remet en évidence la première place qu'occupe le financement des activités au sein des préoccupations des artisans. Il est néanmoins remarquable que non seulement $57,52 \%$ des ressources soient détournées vers d'autres objectifs, mais surtout que $25,15 \%$ d'elles soient consacrées à des activités non productives, telles que les cérémonies et les fêtes. La part destinée aux terrains et constructions n'est pas négligeable non plus (16,05\%). Mais si l'on retient que les logements participent à la formation brute du capital fixe d'une économie $^{11}$, cette troisième destination reste globalement acceptable. Ceci reste valable pour la quatrième $(6,55 \%)$, qui est destinée quant à elle à faire face aux dettes antérieures de l'activité artisanale. C'est dire que, même s'il y a détournement des ressources tontinières vers d'autres fins que la production artisanale, l'utilisation de ces ressources reste globalement acceptable en dehors de la «fausse note " que constituent les dépenses de cérémonies et fêtes. Un nouveau regroupement peut aboutir à la répartition que voici :

Dépenses productives

$51,23 \%$

- Financement artisanat

- Financement autres activités $42,48 \%$

- Remboursement dettes artisanat $6,57 \%$

Dépenses peu productives $2,18 \%$

- Thésaurisation et autres tontines $22,29 \%$

- Terrains et constructions $3,27 \%$

- Remboursement autres dettes $16,05 \%$

- Santé et éducation

11. M. Lutfalla (1981, p. 12). 
Dépenses non productives ${ }^{12}$

$26,48 \%$

- Alimentation

$1,33 \%$

- Cérémonies et fêtes

$25,15 \%$

TOTAL

$100 \%$

Cette dernière répartition montre en effet que les activités financées par les ressources tontinières des artisans sont globalement productives à 73,52\%.

La preuve est faite que l'artisanat béninois a recours aux ressources tontinières pour financer une partie de ses activités. Nos investigations nous permettent en effet de dégager les tendances principales suivantes en matière d'utilisation de ces ressources :

- une source de financement sur trois est d'origine tontinière ;

- presque toutes les entreprises artisanales $(79,25 \%)$ ont recours aux tontines pour financer leurs activités au Bénin ${ }^{13}$;

- un peu plus du quart du financement $(25,17 \%)$ de l'artisanat béninois est assuré par les ressources tontinières.

Ce pourcentage de $25,17 \%$ ne représente paradoxalement que $42,48 \%$ des ressources nettes tontinières disponibles dans le secteur. Un paradoxe qui semble remettre en cause notre hypothèse de base qui retient que l'épargne informelle tontinière finance en priorité l'activité artisanale fondamentalement privée de l'épargne formelle bancaire. Mais en assimilant les aides interpersonnelles aux racines premières des tontines, ce qui nous permet d'agréger aides et ressources tontinières afin d'obtenir les ressources de solidarité de l'informel, nous pouvons constater que $54,44 \%$ des ressources sont d'origine tontinière au sens large et qu'elles représentent $52,47 \%$ des besoins financiers couverts. Ceci confirme avec quelques réserves notre hypothèse de base : l'informel monétaire finance prioritairement l'informel réel, ce financement s'opérant à travers des canaux précis (avances en cours de cycle, levées en fin de cycle et crédits tontiniers), et suivant des conditions d'accès aux fonds plus ou moins onéreuses.

12. On constate que ces dépenses improductives sont effectuées dans l'ordre suivant : artisans de service $(39,45 \%)$, artisans d'art et de décoration $(24,16 \%)$ et artisans de production $(21,95 \%)$.

13. Rappelons que tous les artisans interrogés participent à une tontine. Le taux de $79,25 \%$ explique donc que $20,75 \%$ des artisans détournent la totalité de leurs ressources tontinières à d'autres fins que la production artisanale. 


\section{Les conditions d'accès aux fonds tontiniers dans l'artisanat béninois : le coût de l'argent dans l'informel monétaire}

Dans la présente phase de l'analyse, nous prendrons essentiellement en compte les ressources tontinières au sens strict du terme et non celles qui incluent les aides interpersonnelles. Voulant en effet mettre en évidence non seulement les canaux d'accès aux fonds tontiniers, mais surtout le coût d'utilisation que suppose chaque canal, et n'ayant aucun renseignement sur les conditions et les délais de remboursement des aides interpersonnelles, nous nous sommes limités aux ressources tontinières normales, à partir des deux axes fondamentaux que sont le «portefeuille tontinier » et le coût financier y afférent, supposé/supporté par les entreprises artisanales.

\subsection{Le « portefeuille tontinier » des entreprises béninoises}

\subsubsection{Les éléments constitutifs d'un portefeuille tontinier}

Les sortes (ou nature) de tontines que comporte un portefeuille sont la tontine mutuelle et/ou la tontine commerciale, composantes essentielles du système tontinier béninois. Nos recherches antérieures ont largement présenté les différents aspects de ces deux tontines ${ }^{14}$. Nous nous contenterons d'en présenter les aspects essentiels par rapport à la préoccupation du coût de l'argent qui est la nôtre ici.

\section{Les tontines mutuelles ${ }^{15}$}

Elles sont les plus répandues $(61,04 \%)$ dans le monde des artisans. Ce sont les associations rotatives d'épargne et de crédit (AREC) dans lesquelles les adhérents lèvent à tour de rôle les fonds qu'ils ont préalablement cotisés.

On distingue plusieurs variantes dont les principales sont :

- la tontine mutuelle simple (TMS);

- la tontine mutuelle avec caisse d'entraide (TMCE);

- la tontine mutuelle avec caisse de crédit (TMCC);

14. C.S. Gnansounou (1991a), (1990), (1989).

15. Voir détails dans C.S. Gnansounou (1991a), p. 8-26. 
- la tontine mutuelle avec caisse d'entraide et de crédit (TMEC);

- la tontine à enchères externes (TEE) ;

- la tontine à enchères internes (TEI).

Comme nous le verrons, le coût à supporter dans les tontines mutuelles dépend de la variante dans laquelle on se situe.

\section{Les tontines commerciales ${ }^{16}$}

Les tontines commerciales, quant à elles, semblent moins utilisées que les tontines mutuelles $(38,96 \%$ contre $61,04 \%)$, et sont légèrement moins répandues qu'elles légalement. Elles sont pratiquées dans des associations cumulatrices d'épargne et de crédit (ACEC). Mais il s'agit ici, beaucoup plus de "duos tontiniers » que d'associations tontinières. Une ACEC est en fait une agrégation de plusieurs duos «Tontinier-clients » ayant en commun un même tontinier. Tout se passe comme dans une banque où la banquier est en relation isolément avec chacun de ses clients. Mais comme il arrive souvent dans les tontines commerciales que le noyau, c'est-à-dire le tontinier, invite ses clients à des réjouissances communes, et arrive parfois avec ces mêmes clients à animer des tontines mutuelles, la terminologie « association » peut être également retenue pour les tontines commerciales.

À l'instar des tontines mutuelles, les tontines commerciales comprennent plusieurs variantes dont les principales sont :

- la tontine commerciale à cotisations journalières (TCCJ) ;

- la tontine « Jours du marché » (TJM) ;

- la tontine « Camerounaise » (TC);

- la tontine à cotisations mensuelles progressives (TCMP).

Comme dans les tontines mutuelles, le coût à supporter dans les tontines commerciales dépend de la variante choisie pour diversifier son «portefeuille tontinier ».

\subsubsection{Les " portefeuilles tontiniers"}

À partir de nos enquêtes et suivant la fréquence observée, nous subdiviserons les portefeuilles tontiniers en trois groupes : les portefeuilles à une tontine, les portefeuilles à deux tontines et les portefeuilles à plus de deux tontines.

16. Voir détails dans C.S. Gnansounou (1991a), p. 27-54. 


\section{Les portefeuilles à une tontine $\left(P_{1}\right)$}

Étant donné qu'on peut constater à partir du graphique 1 que les artisans béninois optent majoritairement pour le portefeuille à une tontine $(74,39 \%)$ avec une préférence accentuée pour la tontine mutuelle $(51,42 \%$ contre $22,97 \%$ pour la tontine commerciale), on peut retenir que :

$$
\mathrm{P} 1\left\{\begin{array}{cc}
\mathrm{PT}_{\mathrm{i}}=(1 \mathrm{M}+0) & 51,42 \% \\
\mathrm{ou} & 22,97 \% \\
\mathrm{PT}_{\mathrm{i}}=(0+1 \mathrm{C}) & 74,39 \% \\
\text { TOTAL } &
\end{array}\right.
$$

Cette tendance très prononcée pour le portefeuille à tontine unique doit cependant être prise avec un peu de réserve. La plupart des artisans en effet, qui n'ont déclaré qu'une seule tontine, participent en fait à une autre ${ }^{17}$ qu'ils jugent $^{\prime}$ secondaire et sans importance par rapport à leur activité artisanale et refusent de la faire recenser. C'est pourquoi nous pensons que le choix de portefeuille sera à terme, les portefeuilles à deux tontines.

\section{Les portefeuilles à deux tontines $\left(\mathrm{P}_{2}\right)$}

En ce qui concerne les portefeuilles tontiniers à deux tontines, on a :

$$
\mathrm{P}_{2}\left\{\begin{array}{lr}
\mathrm{PT}_{\mathrm{i}}=(1 \mathrm{M}+1 \mathrm{C}) & 18,21 \% \\
\text { ou } & \\
\mathrm{PT}_{\mathrm{i}}=(2 \mathrm{M}+0) & 2,90 \% \\
\text { ou } & 3,02 \% \\
\mathrm{PT}_{\mathrm{i}}=(0+2 \mathrm{C}) & \\
\quad \text { TOTAL } & 24,13 \%
\end{array}\right.
$$

Ici, chaque portefeuille tontinier comporte deux tontines de même nature $(5,92 \%)$ ou de nature différente $(18,21 \%)$. La forme $\left[\mathrm{PT}_{\mathrm{i}}=(1 \mathrm{M}+1 \mathrm{C})\right]$ représente le portefeuille diversifié le plus utilisé par les artisans et semble être l'une des options les plus retenues pour faire appel aux ressources tontinières, contrairement aux portefeuilles de plus de deux tontines (graphique 1).

17. La tontine non déclarée est souvent qualifiée de «tontine bricole » par les artisans. Ils l'utilisent soit pour financer la tontine qu'ils jugent principale, soit pour financer des projets autres que l'activité artisanale. 
GRAPHIQUE 1

Structure des portefeuilles tontiniers dans l'artisanat béninois

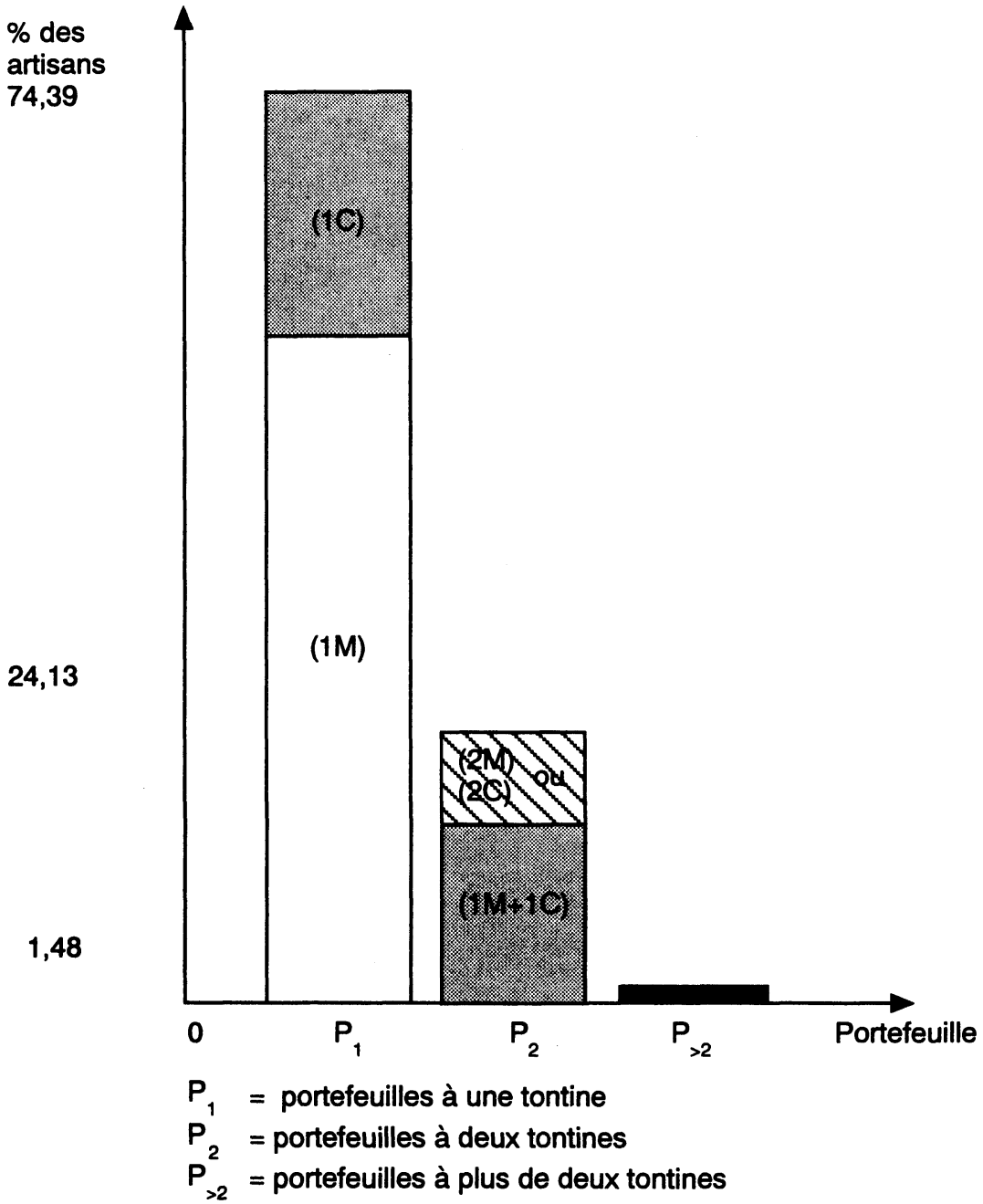

Les portefeuilles à plus de deux tontines $(\mathrm{P}>2)$

Les cas rencontrés $(1,48 \%)$ montrent qu'un artisan peut avoir recours jusqu'à six tontines à la fois. Mais il s'agit d'une tendance naissante qui semble représenter beaucoup plus l'exception que la règle courante de choix de portefeuille tontinier dans le monde artisanal, en vue de l'exploitation de tel ou tel canal d'accès aux fonds. 


\subsection{Les principaux canaux d'accès aux ressources tontinières : les avances, les levées et leur coût}

\subsubsection{Les avances tontinières et les levées de fonds}

Dans les tontines simples sans caisse de crédit, les avances et les levées constituent les deux sources essentielles d'obtention des fonds. En effet, la « levée de fonds » (L) ou le « ramassage » dans toute tontine, permet à chaque adhérent d'entrer dans la totalité des fonds qu'il a cotisés (MBR), déduction faite des crédits sans intérêt appelés avances (A) accordées par le tontinier. Tout ramassage de fonds se fait donc suivant la relation :

$$
\begin{aligned}
& \mathrm{L}=\mathrm{MBR}-\mathrm{A} \\
& \text { avec } \\
& \mathrm{A} \geq 0
\end{aligned}
$$

[A = 0] signifiant que l'artisan n'a pas bénéficié de crédit sans intérêt de la part de sa tontine. Le cas le plus intéressant à étudier ici est celui où $\mathrm{A}$ est supérieur à zéro, c'est-à-dire la possibilité du recours aux avances pour le préfinancement de l'activité artisanale.

Il faut signaler d'entrée de jeu que la pratique des avances tontinières n'est pas propre à une tontine donnée; elle se fait aussi bien dans la mutuelle que dans la commerciale. Mais la décision et les modalités de sa mise en œuvre varie suivant les associations, qu'elles soient rotatives ou accumulatrices, chaque association fixant ses conditions. Ces conditions, plus ou moins souples, sont nécessairement prises en compte par l'artisan au moment de la constitution de son «portefeuille tontinier» s'il privilégie plus ou moins le rôle du préfinancement dans son activité. L'artisan qui considère prioritairement la tontine comme une source de préfinancement $(\mathrm{A} / \mathrm{L}>1)$, et non comme un moyen d'épargne $(\mathrm{A} / \mathrm{L}<1)$, va intégrer dans son portefeuille les tontines à conditions favorables pour les avances, et vice versa. Les deux tendances ont cours d'une façon globalement identique avec les valeurs suivantes du ratio $(\mathrm{A} / \mathrm{L})$ d'utilisation des avances tontinières :

- Artisans de production $\quad 0,98$

- Artisans de service $\quad 1,46$

- Artisans d'art et de décoration $\quad 0,35$

Ensemble

Ces ratios montrent que les artisans de production utilisent d'une manière presque équilibrée les capacités de préfinancement et d'épargne des tontines $(\mathrm{A} / \mathrm{L} \simeq 1)$, que les artisans de service préfèrent disposer avant échéance de leurs 
ressources tontinières, reléguant au second plan les capacités d'épargne des tontines et ce, à l'opposé des artisans d'art et de décoration.

Tenant compte de la structure de dépense à partir des ressources tontinières pour la production artisanale, les ratios $(\mathrm{A} / \mathrm{L})$ se relèvent et mettent beaucoup plus en évidence la préférence des artisans pour les avances tontinières :

- Artisans de production

- Artisans de service

- Artisans d'art et de décoration

Ensemble

Cette tendance au préfinancement est désormais pris en compte dans le « marketing » des tontiniers qui proposent de plus en plus des tontines à conditions très favorables à l'obtention des avances ${ }^{18}$. Nos investigations ont montré que $35,44 \%$ des tontines fréquentées possèdent un système de "levées anticipées » en faveur de leurs membres. Que coûtent donc aux artisans ces levées tontinières, qu'elles soient des « levées à terme » ou des « levées anticipées »?

\subsubsection{Le coût des "levées tontinières":} la commission du tontinier et les pénalités de retard

Dans une tontine classique, les frais que supporte généralement un membre entre sa première cotisation et la levée de fonds se décomposent en frais fixes et frais variables.

Les frais fixes sont les frais de garde (FG) de l'argent prélevés par le tontinier sur toute levée. Il s'agit de la commission du tontinier, fixe dans chaque tontine, et qui varie ici globalement entre $4,26 \%$ et $7,26 \%$ par rapport au montant brut à ramasser (MBR).

Cette commission du tontinier, communément appelée «SOVI » est un don traditionnellement fait au chef tontinier, le "SOGAN », pour le remercier d'avoir gardé les fonds sans les dépenser ${ }^{19}$. Elle est systématiquement prélevée par tous les tontiniers de métier même si cette pratique tend à disparaître dans les milieux intellectuels. Elle représente souvent dans les associations rotatives un peu moins du montant d'un appointement (a), et dans les associations

18. M. Lelart et C. Gnansounou (1989).

19. Pour obtenir des précisions, voir dans C. Gnansounou (1991a, p. 14). 
cumulatrices à peu près le montant d'un appointement. On peut retenir approximativement :

$$
\mathrm{FG} \simeq \mathrm{a}
$$

À côté des frais fixes que constituent les frais de garde, il y a des frais variables qui sont les pénalités de retard que supporte tout adhérent ne respectant pas rigoureusement les délais de cotisations. Fixes à chaque versement dans une tontine donnée, elles se rencontrent surtout dans les associations rotatives et représentent en moyenne le quart de l'appointement à réaliser. Si on les'considère comme variables, c'est que le montant final de pénalités supporté par un adhérent d'une tontine mutuelle dépend du nombre (w) de ses retards au cours d'un cycle tontinier. Il aura à verser au chef de la tontine, le montant

$$
P R \cong w\left(\frac{a}{4}\right), \operatorname{avec} w=0,1,2, \ldots
$$

Le coût total $\left(C_{i}\right)$ à supporter donc, par un artisan (i) avant de lever les fonds qu'il a cotisés équivaut à la rémunération brute tontinière (RBTT) qu'il a consentie d'une manière ou d'une autre au tontinier.

Soit :

$$
\begin{gathered}
\mathrm{C}_{\mathrm{i}}=\underset{\mathrm{RBTT}}{\mathrm{R}}=\mathrm{FG}+\mathrm{PR}_{\mathrm{i}} \\
\mathrm{Cu}_{\mathrm{i}}=\mathrm{a}+\mathrm{w}_{\mathrm{i}}\left(\frac{\mathrm{a}}{4}\right) \\
\text { ou } \\
\mathrm{C}_{\mathrm{i}}=\mathrm{a}\left(\frac{\mathrm{w}_{\mathrm{i}}}{4}\right)
\end{gathered}
$$

Ceci équivaut à un taux de rémunération du tontinier équivalent à

$$
\mathrm{TBRi}=\frac{\mathrm{Ci}}{\mathrm{MBRi}}
$$

Si ce coût (en pourcentage) reste relativement bas dans l'artisanat de production $(4,26 \%)$ et de service $(4,73 \%)$, il est assez significatif dans l'artisanat d'art et de décoration $(7,26 \%)$, ce qui implique que les levées tontinières sont pratiquement deux fois plus chères dans l'artisanat $d$ 'art et de décoration que dans les autres secteurs de production artisanale. Cette déduction est à nuancer, du fait que le calcul de ces coûts a fait intervenir la moyenne arithmétique qui reste avant tout une loi des grands nombres. En effet, les artisans d'art et de décoration sont très peu nombreux par rapport aux autres (134 contre 1486). Par conséquent, le calcul du coût supporté par les premiers comporte plus de biais 
que celui des autres en matière d'utilisation des canaux principaux que constituent les levées et les avances ${ }^{20}$. Qu'en est-il des canaux secondaires?

\subsection{Le canal complémentaire d'accès aux ressources : les crédits tontiniers et leur coût}

Si les avances tontinières constituent des emprunts à taux nul, les crédits tontiniers quant à eux sont de véritables prêts accordés aux artisans par les tontiniers moyennant un loyer de l'argent.

\subsubsection{Crédits tontiniers et financement de l'artisanat}

Les artisans béninois utilisent trois canaux tontiniers de crédit :

- les tontines avec système de crédit pour les membres $(10,03 \%)$;

- les tontines avec système de crédit pour les non-membres $(4,92 \%)$ et

- les tontines avec système de crédit pour les membres et les non-membres $(6,22 \%)$.

Les 2074 sources tontinières recensées se répartissent comme suit :

- tontines sans système de crédit ni d'avance (levées à terme) $\ldots \ldots \ldots \ldots \ldots \ldots \ldots \ldots \ldots \ldots . \ldots 00:(43,39 \%)$

- tontines avec système d'avance (levées anticipées) ...735: $(35,44 \%)$

- tontines avec système de crédit $\ldots \ldots \ldots \ldots \ldots \ldots . .439:(21,17 \%)$

d'où le caractère encore complémentaire des crédits tontiniers par rapport aux canaux principaux de recours aux ressources tontinières $(21,17 \%$ contre $78,83 \%$ ). Cette tendance est plus que confirmée par les montants de ressources mobilisées par les deux canaux :

- levées tontinières $(\mathrm{A}+\mathrm{L}) \ldots \ldots 1660,2$ millions de FCFA $(82,13 \%)$

- crédits tontiniers (CR) …....361,3 millions de FCFA (17,87\%) Ensemble $\ldots \ldots \ldots \ldots \ldots \ldots 2021,5$ millions de FCFA $(100 \%)$

Les crédits tontiniers ne représentent donc en définitive que $21,17 \%$ des sources tontinières et n'assurent que $17,87 \%$ des rentrées tontinières brutes, apport qui sera sensiblement modifié à la hausse si l'on tient compte du coût net de ces crédits (intérêts créditeurs et débiteurs).

20. Remarquons que $43,39 \%$ des sources tontinières ne possèdent ni système d'avance ni système de crédit. 


\subsubsection{Les raisons du succès grandissant des crédits tontiniers}

Le succès relatif des crédits tontiniers auprès des artisans s'explique essentiellement par deux réalités béninoises :

\section{La position de monopole des tontiniers dans la finance informelle}

Le besoin de préfinancement de l'artisanat non satisfait par les institutions financières officielles n'a que la finance informelle pour s'assouvir, ce qui place les détenteurs des crédits tontiniers en situation de monopole.

\section{Le non-respect généralisé des délais de remboursement des crédits tontiniers}

La présente enquête donne un éclairage sur la signification du taux d'intérêt tontinier par rapport à la variable temps. En effet, si certains taux d'intérêt annoncés par les artisans paraissent très élevés, c'est qu'ils ne sont que des taux fictifs ne correspondant en rien aux mécanismes financiers effectivement vécus par eux. Pour mettre en évidence les taux effectifs, il nous faut revenir sur le déroulement de la collecte des informations relatives aux taux d'intérêt. À propos du taux d'intérêt prêteur, par exemple, l'artisan se contente de déclarer à l'enquêteur que «s'il reçoit un crédit de (A) francs CFA, il est virtuellement tenu de rembourser $(A+x)$ francs CFA à la fin du mois ou au bout de deux mois, de trois au plus ». Si c'est le mois qui est la durée retenue, la langage populaire béninois retient dans ce cas : « (A) francs CFA “ enfantent " (x) francs CFA en un mois ».

C'est la traduction financière de ces assertions populaires qui nous a permis de déterminer les taux d'intérêt annuels (i) correspondants, et ce à partir de la formule :

$$
\mathrm{i}=\left(1+\mathrm{i}_{\mathrm{n}}\right)^{\mathrm{n}}-1
$$

avec

$$
\mathrm{n}=\text { le nombre de fois que l'année renferme la durée (d) du crédit }
$$
ainsi

$$
\begin{aligned}
& \mathrm{d}=1 \text { mois }=>\mathrm{n}=12 \\
& \mathrm{~d}=2 \text { mois }=>\mathrm{n}=6 \\
& \mathrm{~d}=3 \text { mois }=>\mathrm{n}=4
\end{aligned}
$$

Ainsi de $10 \%$ mensuel on est passé à $213,84 \%$ pour l'année. Or, la pratique des remboursements montre que le respect de la durée n'est pas du tout strict, et certains ne remboursent en fait leur dette qu'à la fin du cycle de production et de distribution de leurs marchandises, c'est-à-dire au moment qui 
leur semble financièrement favorable et ce, en ne supportant que le taux d'intérêt $\left(i_{n}\right)$ et non le taux annuel. Il faut toutefois remarquer que la pratique des pénalités, qui s'installe de plus en plus, a non seulement pour résultat d'augmenter l'intérêt $(x)$ à payer, mais limite dans une certaine mesure les défaillances dans les délais de remboursement. La tendance reste globalement à la non-capitalisation des intérêts ${ }^{21}$.

Ce constat change totalement les données du problème et montre que le montant de l'intérêt supporté ne dépend pas directement de la durée de l'emprunt. Une telle indifférence quant au facteur temps dans la détermination du taux d'intérêt effectif explique donc en partie le succès des crédits tontiniers qui n'ont plus le coût excessif initialement évalué.

\section{Conclusion}

La présente étude, loin d'aboutir à une recherche visant à relever toutes les difficultés de l'artisanat béninois afin d'y apporter des solutions, reste avant tout un simple test d'utilisation des ressources tontinières dans une activité productive. Notre préoccupation première est donc la tontine. L'artisanat, ici retenu comme l'activité productive la plus proche du système tontinier, nous a servi d'outil d'observation de l'efficacité de la finance informelle en général et des tontines en particulier. C'est dire que notre choix n'a pas été neutre, tontine et artisanat formant pour nous le couple naturel de financement et d'investissement bénéfique pour l'Afrique en général et le Bénin en particulier, le principe de base étant que « la tontine finance prioritairement l'artisanat et l'artisanat n'a que la tontine pour se financer ».

Au terme de notre analyse, force nous est de reconnaître que cette assertion de base n'est pas totalement vérifiée et mérite d'être nuancée.

Les données recueillies et analysées ont mis en évidence que $42,48 \%$ seulement des ressources tontinières mobilisćes ont servi à assurer à peine $25 \%$ des financements réalisés dans l'artisanat béninois, avec la précision que les financements effectivement réalisés ne représentent que $65,55 \%$ des besoins exprimés. Ce qui signifie que non seulement $75 \%$ des besoins réalisés le sont par d'autres ressources que des ressources tontinières, mais que $65,55 \%$ des besoins restent ignorés au moment où $57,52 \%$ des ressources tontinières sont orientés vers d'autres objectifs. Il est par conséquent évident que la tontine ne

21. Ce n'est pas du tout le cas pour les prêts usuraires où la capitalisation est systématique. 
finance pas prioritairement l'artisanat. Mais sachant que les $75 \%$ de besoins non financés par les tontines l'ont largement été par les autres sources informelles de financement, nous retenons que c'est la finance informelle qui finance prioritairement l'artisanat et que l'artisanat n'a que la finance informelle pour se financer.

Toutefois, les ressources tontinières ne sont pas dilapidées pour autant, car à côté des $42,48 \%$ consacrées aux activités artisanales, 31,04\% ont été orientées vers des dépenses plus ou moins productives, portant à 73,52\% la part consacrée à des activités globalement productrices, un chiffre qui rassure en matière d'utilisation bénéfique des ressources tontinières au Bénin.

Mais, au delà de cette assurance, une très grande lacune persiste au niveau des espoirs de plus en plus placés dans les ressources tontinières pour le financement de l'activité productive, de la croissance et du développement dans le tiers monde. Il s'agit du facteur temps qui est décidément utilisé d'une manière très débonnaire dans les crédits tontiniers, enlevant aux ressources tontinières y afférent, une large part de la contrainte de remboursement que sont censés engendrer les fonds d'emprunt dans toute entreprise, contrainte qui impose au chef d'entreprise la gestion rigoureuse et efficiente des moyens financiers dont il dispose.

En effet, si ce « laisser-aller » a l'avantage de fournir des capitaux plus ou moins longs aux entreprises artisanales, il ne favorise pas une gestion rigoureuse des moyens mis en œuvre par les artisans. Il est par conséquent impérieux pour les animateurs de tontines (tontiniers et artisans) de généraliser la formule d'accommodation « cycle de production-distribution » et «cycle tontinier » de manière à créer cette contrainte indispensable à toute gestion efficiente. C'est le lieu de rappeler ce que disait le leader paysan sénégalais Mamadou Cissokho, cité par Fernand Vincent (1986, p. III), « pour que le développement soit local, il faut que la force de développement soit locale... Ce n'est pas normal qu'il y ait toujours des gens pour demander et d'autres pour donner... Le système actuel donateurs-récepteurs n'est pas éternel. Pour que nous soyons indépendants, il nous faut un moyen puissant de financement, d'organisation et de gestion rigoureuse ». Pourquoi pas la tontine? 


\section{Bibliographie}

Babalola, E.J. (1988), «Vie commerciale et socio-économique des districts et de la province de l'Ouémé : le commerce frontalier », Ministère béninois du Commerce, de l'Artisanat et du Tourisme, DPCAT-OUEME, octobre.

Babalola, E.J. (1989), «Les activités de transition et le secteur informel dans la province de l'Ouémé et au Bénin ", Ministère béninois du Commerce, de l'Artisanat et du Tourisme, (MCAT), DPCAT - OUEME, avril-juillet.

Bekolo-Ebe, B et R. Bilongo (1991), "Comportements de gains et structure des taux d'intérêt dans les tontines : étude de quelques cas », dans G. Hénault et R. M'Rabet (1991), p. 107-113.

BIT-PNUD, Projet BEN/86/005 (1987), «Appui aux petits producteurs urbains par la promotion de groupements autogérés », Protocole d'accord entre le Bénin, le PNUD et le BIT préparé par J.P. Lachaud en 1985 et signé le 12 août 1987 à Cotonou.

Brenner, A., H. Fouda et J.M. Toulouse (1990), « Les entrepreneurs Bamilékés de Douala et leur entreprise », Notes de recherche du Réseau entrepreneuriat de l'UREF, $n^{\circ}$ 90-6.

Coulaud, A., C. Croce et B. Dervaux (1986), Les ratios de productivité, Paris, Les Éditions d'Organisation.

Direction de l'Artisanat (1986), « Pourquoi le regroupement professionnel des artisans en République Populaire du Bénin », L'Indicateur, $\mathrm{n}^{\circ} 1$, mars, p. 22.

Direction Provinciale du Commerce de l'Artisanat et du Tourisme du Zou (1988), «Enquête sur la vie commerciale de la province du Zou », sous la direction de Adjibade, AVF, Ministère béninois du Commerce, de l'Artisanat et du Tourisme.

Gero Amoussouga, F.M. (1986), « Place des marchés financiers non organisés dans le développement de la Communauté économique des états de l'Afrique de l'Ouest Référence spéciale au cas des tontines ", $4^{\mathrm{e}}$ Conférence biennale de l'Association des économistes de l'Afrique de l'Ouest, novembre.

Gnansounou, C.S. (1989), « Endettement et financement autonome dans l'économie sousdéveloppée, de nouveaux arguments en faveur de l'épargne intérieure : finance formelle et informelle au Bénin », Thèse de doctorat, Université d'Orléans, mars.

Gnansounou, C.S. (1990), «L'analyse socio-financière des tontines béninoises », CREDO-ENA, Cotonou, août, 220 p.

Gnansounou, C.S. (1991a), «Les tontines, base de l'épargne informelle au Bénin », Institut orléanais de finance, février, $64 \mathrm{p}$.

Gnansounou, C.S. (1991b), «Un secteur productif informel utilisateur des ressources tontinières : l'artisanat béninois ", Institut orléanais de finance, avril $157 \mathrm{p}$. 
Haudeville, B. (1991), «Épargne informelle et financement de l'entreprise productive », dans G. Henault et R. M'Rabet (1991), L'entrepreneuriat en Afrique francophone : culture, financement et développement, Paris, John Libbey Eurotext, p. $77-85$.

Henault, G. et R. M'RABEt (sous la direction de ) (1991), L'entrepreneuriat en Afrique francophone : culture, financement et développement, Paris, John Libbey Eurotext, $328 \mathrm{p}$.

HokPo, L. (1989), «Étude sur la vie commerciale des districts et de la province du Borgou ", Ministère béninois du Commerce, de l'Artisanat et du Tourisme, DPCAT-Borgou, février.

Hugon, Ph. (sous la direction de ) (1980), «Secteur informel et petite production marchande dans les villes du Tiers Monde ", Revue Tiers Monde, vol. XXI, $n^{\circ} 82$, avril-juin.

Hugon, Ph. (1990), « La finance non-institutionnelle : expression de la crise du développement ou de nouvelles formes de développement ? " dans M. Lelart, " La tontine... ", UREF, collection Sciences en marche, Paris, John Libbey Eurotext, p. 309-321.

LACHAUD, J.P. (1986), «Les activités informelles de production au Bénin : analyse et stratégie de développement », Rapport au gouvernement du Bénin, BIT, Genève.

Lelart, M. et C.S. Gnansounou (1989), « Tontines et tontiniers sur les marchés africains : le marché Saint-Michel de Cotonou », African Review of Money Finance and Banking, vol. 1, p. 69-90.

LelaRT, M. (sous la direction de) (1990), La tontine, pratique informelle d'épargne et de crédit dans les pays en voie de développement, UREF, collection Sciences en marche, Paris, John Libbey Eurotext, 356 p.

Lelart, M. (1991), «Les tontines et le financement de l'entreprise informelle », Notes de recherche du réseau entrepreneurial de l'UREF, $\mathrm{n}^{\circ}$ 91-18.

LESPÈs, J.L. (1990), « Les informalités tontinières : traditions et innovations », dans M. Lelart, (1990), op. cit., p. 323-346.

Light, I.S. (1972), Ethnic Enterprise in America : Business and Welfare Among Chinese, Japanese and Blacks, Berkeley, University of California Press.

Lutfalla, M. (1981), «Éléments d'économie », Collection CESB, La Revue Banque Éditeur.

Martineau, J.C. (1991a), « Secteur informel : petit à petit... », Jeune Afrique Économie, $n^{\circ} 141$, mars, p. 113-115.

MARTINEAU, J.C. (1991b), « Secteur informel : s'unir pour survivre ", Jeune Afrique Économie, $\mathrm{n}^{\circ} 141$, mars, p. 115-117. 
Mensah, N. (1991), «Secteur informel : la banque des ferblantiers », Jeune Afrique Économie, $\mathrm{n}^{\circ} 141$, mars, p. 118.

Ministère du Plan et de la Statistique du Bénin (1987), «Avant-projet du plan national de développement économique et social » (1988-1992), Document central, Cotonou, décembre.

Ministère du Plan et DE LA STATISTIQUe du BÉNIN (1987), « Avant-projet du plan national de développement économique et social » (1988-1992) - Secteur industrie et artisanat, Cotonou, décembre.

Miras, C. (1990), «État de l'informel. Informel et état... », Revue Tiers Monde, Tome $\mathrm{XXXI}, \mathrm{n}^{\circ} 122$, avril-juin, p. 377-391.

MotruRA, P. (1977), «Quelques considérations sur la politique des taux d'intérêt dans les pays en voie de développement », Savings and Development, $n^{\circ} 3$, p. 129-149

Mourgues, N. (1990), « Réflexions sur les mécanismes financiers des systèmes tontiniers », dans M. Lelart, (1990), op. cit., p. 245-266.

Nana Sinkam, S.C. (1989), "Structure et rôle des banques en Afrique », Le Courrier $A C P-C E E, \mathrm{n}^{\circ} 117$, septembre-octobre, p. 61-67.

NowaK, M. (1989), «Le financement de l'économie traditionnelle en Afrique », Le Courrier ACP-CEE, $\mathrm{n}^{\circ} 117$, septembre-octobre, p. 68-71.

Oudin, X. (1991), « Dynamique de l'investissement dans le secteur informel : une étude de cas au Niger », dans G. Henault, et R. M'Rabet, (1991), op. cit., p. 145-155.

Pairault, T. (1990), « Sociétés de tontines et banques des petites et moyennes entreprises à Taiwan », dans M. Lelart, (1990), op. cit., p. 281-308.

Présidence de la République Populaire du Bénin (1988), « Déclaration de la stratégie de développement dans le contexte d'un programme d'ajustement structurel », négociation du 28-11-88, Bénin-FMI-Banque mondiale.

Roux, D. 91987), Analyse économique et gestion de l'entreprise, Tome 1, Paris, Dunod, p. 66.

SERVET, J.M. (1990), « Les tontines, formes d'activités informelles et d'initiatives collectives privées en Afrique », dans M. Lelart, (1990), op. cit., p. 267-279.

Tenenbaum, S. (1986), «Immigrants and capital : Jewish loan societies in the United States, 1880-1940», Thèse de doctorat, Brandeis University.

VINCENT, F. (1986), Manuel de gestion pratique des associations de développement rural du Tiers Monde, Tome II, IRED, Genève. 